\title{
Relação entre fatores ambientais e qualidade de vida em indivíduos com acidente vascular cerebral usuários da atenção primária do Sistema Único de Saúde
}

\section{Relationship between environmental factors and quality of life in post stroke users of primary health care of the Unified Health System}

Camila Ferreira da $\mathrm{Cruz}^{1}$, Soraia Micaela Silva ${ }^{2}$, Erika de Freitas Araújo ${ }^{1}$, Paula da Cruz Peniche ${ }^{1}$, Larissa Tavares Aguiar ${ }^{1}$, (DChristina Danielli Coelho de Morais Faria ${ }^{1}$

\section{RESUMO}

O fator ambiental constitui o ambiente físico, social e atitudinal em que indivíduo vive e conduz sua vida, incluindo barreiras e/ou facilitadores. A qualidade de vida é um construto amplo que envolve vários fatores, dentre eles o ambiental. A relação entre qualidade de vida relacionada à saúde (QVRS) após Acidente Vascular Cerebral (AVC) e fatores ambientais ainda é pouco explorada. Conhecer essa relação pode facilitar o processo de avaliação e planejamento de intervenções para melhora da QVRS desses indivíduos. Objetivo: Investigar a associação entre fatores ambientais e QVRS de indivíduos com AVC, na fase crônica, usuários da atenção primária à saúde de Belo Horizonte (BH)/Brasil. Métodos: Fatores ambientais foram avaliados pelo Measure of the Quality of Environment (MQE) e a QVRS pela Escala de Qualidade de Vida Específica para Acidente Vascular Encefálico (EQVE-AVE). Correlação de Spearman foi utilizada para investigar a associação entre fatores ambientais e QVRS (escore total e dos domínios do EQVE-AVE) ( $\alpha=5 \%$ ). Resultados: Foram encontradas associações significantes, negativas e de fraca magnitude entre fatores ambientais considerados como obstáculos com o escore total do EQVE-AVE $(p=0,001 ;$ rho $=-0,33)$ e com os domínios de mobilidade $(p=0,01$ rho $=-0,33)$ e trabalho/produtividade do EQVE-AVE $(p=0,03$; rho $=-0,28)$. Conclusão: Fatores ambientais e QVRS possuem associação significante, ainda que seja de fraca magnitude. Portanto, fatores ambientais considerados como obstáculos, devem ser considerados na abordagem da QVRS de indivíduos na fase crônica após AVC, usuários da atenção primária à saúde do Brasil.

Palavras-chave: Acidente Vascular Cerebral, Qualidade de Vida, Classificação Internacional de Funcionalidade, Incapacidade e Saúde

1 Programa de Pós-graduação em Ciências da Reabilitação, Universidade Federal de Minas Gerais - UFMG

2 Programa de Pós-graduação em Ciências da Reabilitação, Universidade Nove de Julho UNINOVE

\section{Correspondência}

Christina Danielli Coelho de Morais Faria

E-mail: cdcmf@ufmg.br

Submetido: 26 Dezembro 2019

Aceito: 06 Fevereiro 2020.

\section{Como Citar}

Cruz CF, Silva SM, Araújo EF, Peniche PC, Aguiar LT, Faria CDCM. Relação entre fatores ambientais e qualidade de vida em indivíduos com acidente vascular cerebral usuários da atenção primária do Sistema Único de Saúde. Acta Fisiatr. 2019;26(2):108-114.

DOI: 10.11606/issn.2317-0190.v26i2a166378

\section{cc) $\$($}

\section{ABSTRACT}

Quality of life is a broad and comprehensive construct that involves several factors, including the environmental factor. However, the relationship between health-related quality of life (HRQoL) of individuals after stroke and environmental factors is still poorly understood. Knowing this relationship can facilitate the process of evaluating and planning interventions to improve HRQoL of these individuals. Objective: To investigate the association between environmental factors and HRQoL of individuals after stroke who use the primary health care in Belo Horizonte/Brazil. Methods: Environmental factors were assessed by the Measure of the Quality of Environment (MQE) and the HRQoL by the Stroke Specific Quality of Life Scale (SS-QOL). Spearman's correlation was used to investigate the association between environmental factors and HRQoL (total score and SS-QOL domains) ( $\alpha=5 \%$ ). Results: Significant, negative and low associations were found between environmental factors considered as obstacles with the total SS-QOL score $(p=0.001$; rho $=-0.33)$, with the mobility $(p=0.01$ rho $=-0.33)$ and work/productivity domains of the HRQoL $(p=0.03$; rho = -0.28). Conclusion: Therefore, environmental factors and HRQoL have a significant association, even if it is of low magnitude. Thus, environmental factors considered as obstacles, should be considered, along with other factors related to health and functioning when addressing the HRQoL of individuals in the chronic phase after stroke who use the Brazilian primary health care system.

Keywords: Stroke, Quality of Life, International Classification of Functioning, Disability and Health 


\section{INTRODUÇÃO}

O Acidente Vascular Cerebral (AVC) é uma doença com desenvolvimento rápido de sinais clínicos de distúrbios focais e/ou globais da função cerebral com duração superior a 24 horas. ${ }^{1}$

No Brasil, a prevalência do AVC é de 2.231.0002, gerando incapacidades graves, atingindo mais de 568.000 indivíduos, ${ }^{2}$ com comprometimento em diferentes aspectos da funcionalidade. ${ }^{3}$ Por este motivo, as Diretrizes de atenção à Reabilitação da pessoa com AVC considerada esta condição de saúde como prioridade na saúde pública nacional. ${ }^{4}$

Para o entendimento do complexo processo de funcionalidade e incapacidade humana, a Organização Mundial de Saúde (OMS) propõe a utilização de uma classificação baseada no modelo biopsicossocial que tem sido muito aplicada em indivíduos após AVC: a Classificação Internacional de Funcionalidade, Incapacidade e Saúde (CIF). ${ }^{5}$

Segundo a CIF, saúde e funcionalidade interagem de forma dinâmica e complexa com os fatores contextuais, que englobam os fatores pessoais e ambientais. $O$ fator ambiental constitui o ambiente físico social e atitudinal em que indivíduo vive e conduz sua vida. ${ }^{5}$

Esse componente pode ter influência negativa ou positiva na vida das pessoas de modo a facilitar ou limitar o desempenho de atividades e a participação social. ${ }^{5}$ Dessa maneira, o fator ambiental pode ser visto como barreira ou facilitador para as estruturas e funções corporais, atividade ou participação. ${ }^{5}$

Outros desfechos importantes de saúde, como a qualidade de vida, também podem sofrer impacto negativo com as incapacidades advindas com o AVC e que apresentam potencial de interação com os fatores ambientais. A qualidade de vida é um construto amplo e abrangente e que é influenciada de forma complexa pela saúde física do indivíduo, estado psicológico, nível de independência, relação social, crenças pessoais e sua relação com seu ambiente. ${ }^{6}$

Eventualmente, a qualidade de vida é equiparada com conceitos subjetivos de bem estar ou de satisfação com a vida. ${ }^{7}$ Considerando a multidimensionalidade da qualidade de vida, foi proposto o conceito de Qualidade de Vida Relacionado à Saúde (QVRS). A QVRS é a percepção do indivíduo, ou de um grupo ao longo do tempo, sobre sua doença e seus impactos na própria vida, incluindo a satisfação pessoal associada ao seu bem estar físico, emocional e social. ${ }^{8}$

Algurén et al. ${ }^{9}$ mostraram que a QVRS na fase aguda após AVC parece não estar apenas associada à aspectos do corpo, mas também aos fatores ambientais. ${ }^{9}$ Os autores apontaram neste estudo a necessidade de mais pesquisas para investigar melhor a existência da associação entre fator ambiental e QVRS. ${ }^{9}$

Os resultados de estudos em outras populações acometidas por doenças que também apresentam importante impacto na saúde e funcionalidade podem ilustrar a importância de se investigar melhor essa relação. ${ }^{10,11,12} \mathrm{Um}$ amplo estudo longitudinal, por exemplo, realizado em crianças com paralisia cerebral demonstrou que os fatores ambientais são um dos determinantes da participação e QVRS. ${ }^{10}$

A associação entre fator ambiental e QVRS ainda não foi investigada em indivíduos após AVC da atenção primária do Sistema Único de Saúde (SUS). ${ }^{13}$

A atenção primária é a principal porta de entrada dos usuários do SUS e o centro de comunicação de toda rede de atenção à saúde. ${ }^{13}$ Portanto, investigar essa associação no contexto da atenção primária pode direcionar futuros estudos e ampliar estratégias que visam melhorar a QVRS após AVC.

\section{OBJETIVO}

Investigar a associação entre fatores ambientais e QVRS (escore total e dos domínios do EQVE-AVE) de indivíduos após AVC usuários da atenção primária à saúde de Belo Horizonte/MG.

\section{MÉTODOS}

Trata-se de um estudo transversal exploratório o qual faz parte de um projeto de pesquisa mais amplo, denominado "Perfil de funcionalidade, incapacidade e saúde de indivíduos acometidos pelo Acidente Vascular Cerebral e usuários de Unidades Básicas de Saúde da cidade de Belo Horizonte", aprovado pelo Comitê de Ética em Pesquisa (COEP) da Universidade Federal de Minas Gerais (UFMG) e da Secretária Municipal de Saúde de Belo Horizonte (SMSA-BH) (CAAE:14038313400005149).

Os dados foram coletados em quatro Unidade Básica de Saúde (UBS) localizadas nos distritos sanitários Norte, Nordeste, Centro Sul e Venda Nova de Belo Horizonte/MG. As UBS selecionadas atenderam aos seguintes critérios que são preconizados pelo Ministério da Saúde: presença de Equipe de Saúde da Família (ESF) formada por no mínimo um médico, um enfermeiro, um auxiliar de enfermagem e agentes comunitários de saúde. ${ }^{14}$

Acompanhamento de no máximo quatro mil pessoas de determinado território por parte de cada equipe. ${ }^{14}$ Ademais, as UBS recebem o apoio do Núcleo Ampliado de Saúde da Família (NASF), no que rege a Portaria MS/GM n ${ }^{\circ} 154 / 2008$ a qual institui critérios de número mínimo e máximo de ESF às quais NASF deve estar vinculado e o número de profissionais com nível superior. ${ }^{14,15}$

Inicialmente, foram realizadas visitas às UBS para identificar possíveis indivíduos junto aos profissionais da área da saúde. Em seguida, foram realizadas visitas domiciliares para coletas dos dados. ${ }^{3}$ Para cada indivíduo, a coleta de dados foi completamente realizada com uma única visita domiciliar.

\section{Amostra}

Profissionais da ESF e do NASF das UBS's identificaram indivíduos com AVC usuários do SUS que moravam na região de metropolitana de Belo Horizonte e que atendiam aos seguintes critérios de inclusão: ter diagnóstico médico de AVC com tempo de acometimento superior a seis meses (fase crônica), idade igual ou superior à 20 anos, não possuir outra doença neurológica ou incapacitante diagnosticada.

Todos indivíduos inclusos deveriam ainda, assinar o Termo de Consentimento Livre e Esclarecido (TCLE) aprovado pelo COEP da UFMG e da SMSA-BH.

Os critérios de exclusão foram: provável déficit cognitivo, identificado por meio do questionário Mini Exame do Estado Mental e considerando a classificação com base na escolaridade do indivíduo (13 pontos para analfabetos, 18 para indivíduos com 1 a 7 anos de estudo e 26 para 8 ou mais anos de estudos); afasia motora; ter mudado de cidade; ter sido institucionalizado; ter viajado durante período de coleta; não ter completado a coleta de dados necessária para fornecer os dados utilizados no presente estudo.

\section{Instrumentos de Medida}

QVRS específica para o AVC: Escala de Qualidade de Vida Específica para Acidente Vascular Encefálico (EQVE-AVE).

O EQVE-AVE é um questionário desenvolvido para medir a QVRS específica de indivíduos após AVC. ${ }^{16} \mathrm{O}$ questionário é formado por 12 domínios, com 49 itens no total que estão relacionados a três domínios específicos da CIF: estrutura e função do corpo, atividade e participação. ${ }^{16,17}$

O EQVE-AVE foi traduzido, validado e apresenta adequadas propriedades de medida ${ }^{18}$ e deve ser aplicado sob entrevista.

Para análise dos dados, foram considerados o escore total, que pode variar de 49 a 245 pontos, e os escores dos domínios: "Energia" (3 a 15 pontos); "Papel familiar" (3 a 15 pontos); "Linguagem" (5 a 25 pontos); "Mobilidade" (6 a 30 pontos); "Humor" (5 a 25 pontos); "Personalidade" ( 3 a 15 pontos); "Autocuidado ( 5 a 25 pontos); "Papel social" (5 a 25 pontos); "Raciocínio" (3 a 15 pontos); "Função de 
membro superior" (5 a 25 pontos); "Visão" (3 a 15 pontos) e

"Trabalho/produtividade" ( 3 a 15 pontos). ${ }^{16}$

\section{Fatores ambientais: Measure of the Quality of Environment (MQE)}

O MQE é um questionário, utilizado para a medida da percepção dos indivíduos em relação aos fatores ambientais, se eles são vistos como facilitadores ou barreiras para a realização das atividades de vida diária. ${ }^{19}$ Este instrumento abrange 26 itens, os quais abordam questões sobre o ambiente físico e social do indivíduo e possui uma escala de escore de sete pontos a qual varia de -3 , que significa um obstáculo importante, e +3 , que significa facilitador principal. ${ }^{20}$

Há versões longa e curta para o MQE, ambas contemplando seis domínios que englobam os cinco capítulos do componente Fatores Ambientais da CIF. Os domínios do MQE são: atitudes e suporte social; trabalho e renda; serviços governamentais e públicos; igualdade de oportunidade; orientações políticas, referentes ao ambiente social; por fim estrutura física e acessibilidade e tecnologia, referentes ao ambiente físico. ${ }^{21}$

A vantagem de se utilizar a versão curta do instrumento é pela objetividade, com rápida aplicação e fácil interpretação dos escores. A versão curta do MQE já foi testada em indivíduos com AVC, foi traduzida, validada e demonstrou boa reprodutibilidade. ${ }^{21}$ No presente estudo, foi utilizada a versão curta do MQE, aplicada sob entrevista, conforme recomendado. ${ }^{22}$

\section{Escala de Fugl-Meyer (EFM)}

A EFM avalia o comprometimento sensório-motor da extremidade superior e inferior de indivíduos pós AVC. ${ }^{23}$ A EFM possui valores apropriados de confiabilidade e validade, inclusive na população brasileira foi traduzida e adaptada culturalmente com valores adequados de confiabilidade. ${ }^{24}$ A pontuação máxima considerando ambas extremidades é 100 pontos, sendo divididos em 66 pontos para extremidade superior e 34 para a inferior. ${ }^{24}$

A classificação do comprometimento motor global do indivíduo é conforme a pontuação adquirida, sendo que 50 pontos indica comprometimento motor severo, 50-84 marcante, 85-95 moderado, 96-99 leve e 100 sem comprometimento motor. ${ }^{24}$ Sendo assim, essa será a classificação utilizada no presente estudo.

\section{Procedimentos}

Todos os dados foram coletados por pesquisadores previamente treinados com todos os procedimentos que seriam realizados e com auxílio de outro examinador, também treinado. Os pesquisadores participaram efetivamente de reuniões das ESF e do NASF das UBS com intuito de identificar os indivíduos após AVC que se enquadrassem nos critérios de inclusão já descritos.

Além de identificar os indivíduos elegíveis nas UBS, os pesquisadores coletaram informações no prontuário dos pacientes, após consentimento dos mesmos, para serem confrontadas com o relato do paciente em visita domiciliar.

Foi realizada uma única visita em domicílio para coleta de dados para caracterização da amostra, que envolveu informações sociodemográficas e clínicas, como o grau de comprometimento motor avaliado pela Escala Fugl-Meyer e, para a coleta de dados dos desfechos principais: QVRS (EQVE-AVE) e fatores ambientais (MQE).

Foi orientado aos acompanhantes e/ou cuidadores que não interferissem no momento de aplicação dos testes para evitar erros durante o procedimento.

\section{Análise estatística}

O teste de normalidade Shapiro-Wilk foi utilizado para analisar a distribuição dos dados das variáveis quantitativas. Para as variáveis quantitativas, normalmente distribuídas, foi utilizada a estatística descritiva por meio da média e desvio padrão. Para as variáveis sem distribuição normal, utilizou-se a mediana e o intervalo interquartílico. Frequência foi utilizada para as variáveis categóricas.

O grau de associação entre fatores ambientais e QVRS foi analisado por meio do coeficiente de correlação de Spearman (rho). A força ou magnitude da correlação significativa entre as variáveis foi classificada como fraca (coeficiente de correlação entre $\leq 0,39$ ), moderada (entre 0,4 a 0,69) e forte (entre 0,7 e 1,00). ${ }^{25} \mathrm{Em}$ todas as análises inferenciais foi considerado $\alpha=0,05$ e utilizado o programa SPSS para Windows (versão 19).

\section{RESULTADOS}

Foram identificados 133 indivíduos elegíveis para compor a amostra. Contudo, apenas 63 desses indivíduos foram incluídos por atenderam aos critérios de elegibilidade. O fluxograma (Figura 1) ilustra os motivos de exclusão desses indivíduos.

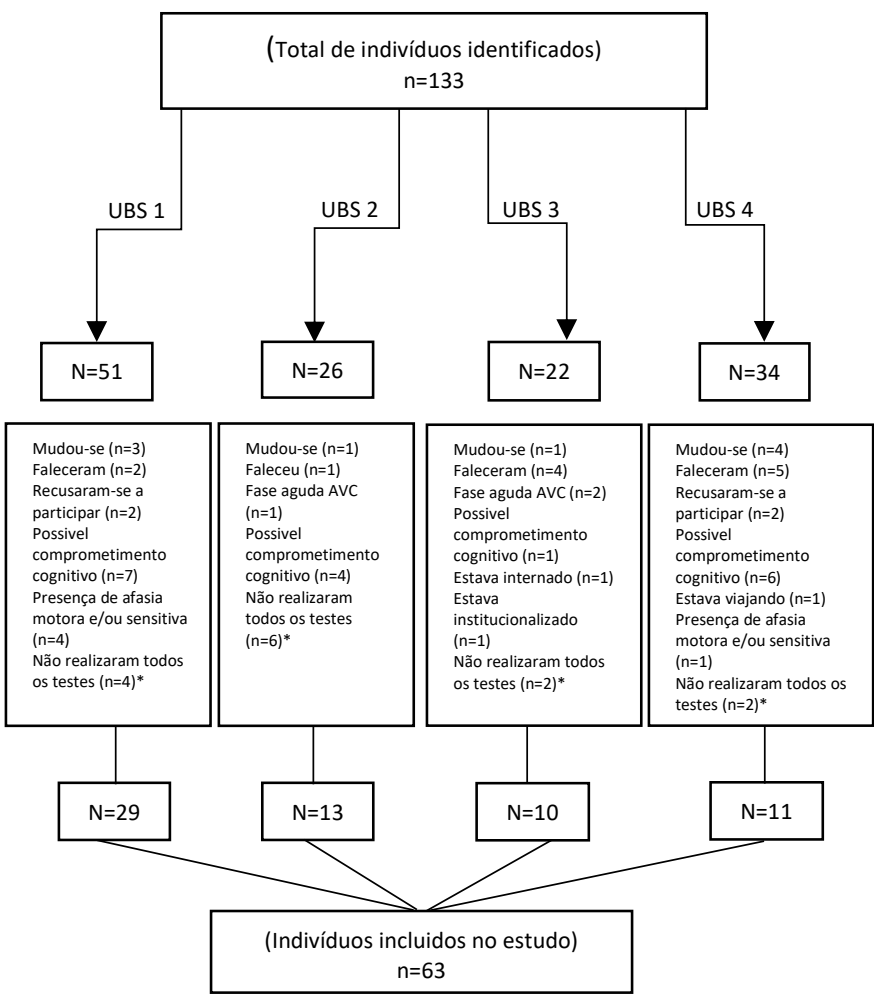

UBS= Unidade Básica de Saúde; $n=$ número de indivíduos; $A V C=$ Acidente vascular cerebral; *Motivos para a não realização dos testes: não conseguir responder a todas as questões dos questionários por estarem fisicamente debilitados

Figura 1. Fluxograma da composição da amostra, com os motivos deexclusão do estudo

A média de idade da amostra analisada foi de $65,8 \pm 12,1$ anos. A maioria dos participantes eram casados $(38,1 \% n=24)$ ou viúvos $(33,3$ $\% \mathrm{n}=21$ ).

A classe socioeconômica mais frequente foi C1 $(38,1 \% n=24)$, seguida de $C 2(30,2 n=19)$. Nenhum indivíduo foi categorizado nas classes A1 e A2.

O nível de escolaridade mais predominante foi ginásio incompleto $(39,7 \% n=25)$, seguido de primário incompleto e não saber ler/escreve $(23,8 \% n=15)$.

O tempo médio de evolução após AVC foi $60,3 \pm 54,2$ meses e a maioria dos indivíduos relataram quatro ou mais doenças associadas $(82,5 \% \mathrm{n}=52)$. Os demais resultados das características sociodemográficas e clínicas dos indivíduos incluídos (Tabela 1). 
Tabela 1. Dados clínicos-demográficos das variáveis do estudo

\begin{tabular}{|c|c|}
\hline Variável & $n=63$ \\
\hline \multicolumn{2}{|l|}{ Sexo-n (\%) } \\
\hline Feminino & $33(47,6)$ \\
\hline Masculino & $30(52,4)$ \\
\hline Idade (anos) - média (DP) & $65,8( \pm 12,1)$ \\
\hline Tempo de Evolução (meses) - média (DP) & $60,3( \pm 54,2)$ \\
\hline \multicolumn{2}{|l|}{ Estado civil - $\mathbf{n}(\%)$} \\
\hline Solteiro & $10(15,9)$ \\
\hline Casado & $25(39,7)$ \\
\hline União Estável & $2(3,2)$ \\
\hline Viúvo & $21(33,3)$ \\
\hline Divorciado & $5(7,9)$ \\
\hline \multicolumn{2}{|l|}{ Escolaridade - $n(\%)$} \\
\hline Não sabe ler/escrever & $15(23,8)$ \\
\hline Primário Incompleto & $15(23,8)$ \\
\hline Ginásio incompleto & $26(41,3)$ \\
\hline Colegial incompleto & $2(3,2)$ \\
\hline Superior incompleto & $5(7,9)$ \\
\hline \multicolumn{2}{|l|}{ Doenças associadas - $\mathrm{n}(\%)$} \\
\hline Uma doença & $1(1,6)$ \\
\hline Duas doenças & $7(11,1)$ \\
\hline Três doenças & $3(4,8)$ \\
\hline Quatro doenças & $52(82,5)$ \\
\hline Quantidade de medicamentos - média (DP) & $4,0( \pm 2,5)$ \\
\hline \multicolumn{2}{|l|}{ Classe Socioeconômica - n (\%) } \\
\hline Classe B1 & $4(6,3)$ \\
\hline Classe B2 & $5(7,9)$ \\
\hline Classe $\mathrm{C} 1$ & $24(38,1)$ \\
\hline Classe C2 & $19(30,2)$ \\
\hline Classe D & $9(14,3)$ \\
\hline Classe E & $1(1,6)$ \\
\hline \multicolumn{2}{|l|}{ Comprometimento motor $\mathrm{n}(\%)$} \\
\hline Severo & $11(17,5)$ \\
\hline Marcante & $21(33,3)$ \\
\hline Moderado & $20(31,7)$ \\
\hline Leve & $8(12,7)$ \\
\hline Sem comprometimento motor & $3(4,8)$ \\
\hline \multicolumn{2}{|l|}{ MQE, mediana (IQ) } \\
\hline Facilitador & $1,3\left(1,8 \_1,1\right)$ \\
\hline Obstáculo & $-2,5(-1,67-2,75)$ \\
\hline Mini Mental, mediana (IQ) & $22\left(26 \_19,8\right)$ \\
\hline EQVE-AVE (escore total) - mediana (IQ) & $174,0(195,0-143,0)$ \\
\hline \multicolumn{2}{|l|}{ Domínios EQVE-AVE - mediana (IQ) } \\
\hline Energia & $8(14-6)$ \\
\hline Papéis Familiares & $7(12-5)$ \\
\hline Linguagem & $21(24-16)$ \\
\hline Mobilidade & $21(27-14)$ \\
\hline Humor & $18(22-12)$ \\
\hline Personalidade & $7(11-4)$ \\
\hline Auto cuidado & $23(25-18)$ \\
\hline Papéis Sociais & $11(15-9)$ \\
\hline Memória Concentração & $12(14-9)$ \\
\hline Função Extremidades Superior & $21(24-14)$ \\
\hline Visão & $14(15-11)$ \\
\hline Trabalho e Produtividade & $11(13-7)$ \\
\hline
\end{tabular}

Legenda: MQE =Measure of the Quality of Environment); EQVE-AVE= Escala de Qualidade de Vida Especifica para Acidente Vascular Encefálico; \%= Porcentagem; $n=$ frequência; $D P=$ Desvio Padrão; $1 Q=$ Intervalo Interquatil

Considerando o escore total do EQVE-AVE, observou-se associação significante, negativa e de fraca magnitude entre fatores ambientais considerados obstáculos e a QVRS $(p=0,001$; rho $=-0,33)$.

Foram observadas, também, associações significantes, negativas e de fraca magnitude entre fatores ambientais considerados obstáculos e os domínios de mobilidade $(p=0,01 ;$ rho $=-0,33)$ e trabalho/produtividade $(p=0,03$; rho $=-0,28)$ do EQVE-AVE.

Não houve correlação significante entre fatores ambientais facilitadores e a QVRS (Tabela 2).

Tabela 2. Associação entre ambiente (MQE) e qualidade de vida (EQVE-AVE)

\begin{tabular}{lcccc}
\hline \multicolumn{1}{c}{$\begin{array}{c}\text { EQVE-AVE total } \\
\text { e domínios }\end{array}$} & \multicolumn{2}{c}{ MQE-OBSTÁCULO } & \multicolumn{2}{c}{ MQE-FACILITADOR } \\
& rho* & $\mathbf{p}^{* *}$ & rho* & p** \\
\hline Total & $-0,33^{* * *}$ & 0,001 & $-0,82$ & 0,52 \\
Energia & $-0,13$ & 0,30 & $-0,81$ & 0,53 \\
Papeis familiares & $-0,25$ & 0,05 & $-0,17$ & 0,17 \\
Linguagem & $-0,14$ & 0,27 & 0,16 & 0,20 \\
Mobilidade & $-0,33^{* * *}$ & 0,01 & $-0,88$ & 0,49 \\
Humor & $-0,23$ & 0,07 & $-0,06$ & 0,62 \\
Personalidade & $-0,17$ & 0,18 & 0,03 & 0,79 \\
Auto cuidado & $-0,21$ & 0,09 & $-0,24$ & 0,06 \\
Papéis sociais & $-0,24$ & 0,06 & $-0,13$ & 0,31 \\
Memória concentração & $-0,09$ & 0,48 & $-0,16$ & 0,22 \\
Função extremidades & $-0,10$ & 0,40 & 0,047 & 0,71 \\
superiores & $-0,21$ & 0,09 & 0,05 & 0,72 \\
Visão & $-0,28 * * *$ & 0,03 & $-0,09$ & 0,48 \\
\hline Trabalho e produtividade & Legenda: ${ }^{*}$ coeficiente de Spearman; ${ }^{* *}$ valor de $p ; * * *$ Variáveis significativas &
\end{tabular}

\section{DISCUSSÃO}

Este estudo teve como objetivo investigar a associação entre fatores ambientais e QVRS de indivíduos com AVC usuários da atenção primária à saúde de Belo Horizonte/MG. Os resultados deste estudo demonstraram que há associação negativa de fraca magnitude entre os fatores ambientais considerados obstáculos e a QVRS, considerando tanto a pontuação total quanto a dos domínios mobilidade e trabalho/produtividade do EQVE-AVE.

Acerca das características sociodemográficas e clínicas dos participantes, observa-se que foram similares a de estudos prévios realizados com indivíduos após AVC na fase crônica, sendo predominante o sexo feminino; ${ }^{26,27}$ maioria idosos; ${ }^{27}$ estado conjugal mais comuns casados ou viúvos; ${ }^{28,29,30}$ com baixo nível de escolaridade e socioeconômico; ${ }^{31,32}$ com três ou mais comorbidades; em uso de muitos medicamentos ${ }^{33,34}$ e com comprometimento motor marcante e moderado. ${ }^{35,36}$

Pereira et al. ${ }^{37}$ desenvolveram também um estudo na atenção primária, porém com foco em educação ambiental abrangendo a maior parte dos usuários da comunidade de Santa Fé (Uberlândia/Minas Gerais (MG), ${ }^{37}$ contudo, não houve objetivo em atingir especificamente indivíduos com AVC, mas sim uma população mais genérica abrangendo fatores determinantes de saúde como um todo. Assim, não conseguimos contrastar as características da amostra avaliada com a de outros usuários do SUS de Minas Gerais.

A associação negativa entre fatores ambientais considerados obstáculos e QVRS demonstra que quanto maior a percepção de obstáculos, menor será o escore referente à QVRS. Resultados semelhantes foram reportados no estudo de Parra et al. ${ }^{38}$ cujo objetivo foi examinar associações entre percepções do ambiente com QVRS de indivíduos idosos na cidade de Bogotá. Foi observado que ruídos na rua, uma das percepções do ambiente, foi um preditor significante da QVRS. ${ }^{38}$

Em outro estudo de Badia et al. ${ }^{39}$ com crianças e adolescentes com paralisia cerebral, foi observado que a falta de adaptações no ambiente domiciliar e escolar está associado a baixa QVRS desses indivíduos. ${ }^{39}$ Além disso, Law et al. ${ }^{40}$ mostraram em seu estudo que a 
QVRS de crianças com alguma incapacidade física é influenciada por barreiras presentes no ambiente. Apesar desses estudos terem sido realizados com população diferente do presente estudo, é possível observar a associação entre os fatores ambientais e QVRS em indivíduos com diferentes condições de saúde.

Yeung \& Tower ${ }^{41}$ realizaram um estudo com base nos conceitos da CIF para investigar a influência dos componentes desta classificação na QVRS de jovens com incapacidades.

Foi realizada uma análise de regressão linear para analisar a influência dos componentes da CIF, incluindo fatores ambientais, como preditores da QVRS. Foi observado que todos componentes da CIF influenciam o nível de QVRS dos indivíduos do estudo, incluindo os fatores ambientais. ${ }^{41}$ Cabe ressaltar, ainda, que não foram encontrados estudos que tenham analisado a associação entre ambiente e QVRS após AVC utilizando o MQE e EQVE-AVE, respectivamente, o que dificulta a comparação dos presentes resultados.

As associações negativas observadas entre ambiente considerado como obstáculo e os domínios mobilidade e trabalho/produtividade do EQVE-AVE podem ser melhor entendida se considerarmos os resultados do estudo Zhao \& Chung, ${ }^{42}$ no qual avaliou-se associações entre atributos do ambiente relacionados à mobilidade com a QVRS de idosos em Hong Kong.

Para tanto, considerou-se como variáveis independentes os riscos no trânsito, a infraestrutura para caminhadas, desnivelamento no solo e barreiras físicas. ${ }^{42}$ As dimensões física e mental da QVRS foram consideradas como variáveis dependentes..$^{42}$

O instrumento utilizado para a análise dos atributos ambientais foi um questionário denominado "Neighbourhood Environment Walkability Scale" (NEWS-A) que leva em consideração todas as variáveis independentes citadas. ${ }^{42}$ Para QVRS foi utilizado o Short Form-36 (SF-36) que abrange os domínios citados para o estudo..$^{42}$ Os autores reportaram associação positiva entre ambiente seguro (como boa infraestrutura, sem barreiras físicas) e a QVRS nos domínios físicos e mentais de idosos em Hong Kong. ${ }^{42}$

Esse achado corrobora com os achados do presente estudo, pois, um ambiente percebido com muitos obstáculos, por exemplo, tem associação com menores pontuações na dimensão física (mobilidade) da QVRS. O mesmo raciocínio se aplica para o domínio trabalho/produtividade, quanto maior percepção de fatores ambientais considerados obstáculos, menores pontuações no domínio trabalho/produtividade do EQVE-AVE.

O estudo de Gobbens \& Assen ${ }^{43}$ também contribui para o melhor entendimento dos achados do presente estudo. Um dos objetivos dos autores foi analisar a associação dos fatores ambientais com a QVRS no domínio físico, psicológico e social em idosos. ${ }^{43}$ Ao término, foram observadas associações entre múltiplos fatores ambientais com a QVRS de idosos. ${ }^{43}$ Os autores relataram, ainda, que a QVRS pode ser otimizada se retirados os obstáculos do ambiente domiciliar. ${ }^{41}$

Em indivíduos na fase crônica após AVC, diversos fatores relacionados à saúde e à funcionalidade já foram significativamente associados à QVRS, como déficit cognitivo, escolaridade, déficit de equilíbrio e sexo. ${ }^{44,45}$ Segundo os resultados do presente estudo, os fatores ambientais considerados obstáculos são outros fatores que devem ser considerados na abordagem da QVRS desses indivíduos.

Ainda neste sentido, Riberto et al. ${ }^{46}$ validaram empiricamente $o$ core set da CIF para o AVC, verificando a frequência de problemas em cada uma de suas categorias, verificando a validade do conteúdo. Ao término do estudo, observaram que todos os fatores ambientais foram qualificados como facilitadores ou barreiras e atuaram como moduladores da deficiência. ${ }^{46}$ Portanto, os fatores ambientais não podem ser negligenciados no processo de avaliação da funcionalidade após AVC.

A atenção primária é caracterizada por um conjunto de ações de saúde que envolvem promoção, prevenção de agravos, diagnóstico e tratamento. ${ }^{47} \mathrm{O}$ estudo atual foi realizado com indivíduos após AVC usuários da atenção primária do SUS. As associações observadas neste estudo contribuem para melhor entendimento da relação entre QVRS e fator ambiental e pode auxiliar na elaboração de futuros estudos e de estratégias de ações na atenção primária do SUS cujo foco seja a QVRS desses indivíduos.

Já foi demonstrado em um estudo prévio desenvolvido com usuários da atenção primária do SUS em cinco regiões do Brasil, que a QVRS é influenciada pelo estilo de vida e fatores sociodemográficos. ${ }^{48}$ Os resultados do presente estudo complementam que os fatores ambientais também apresentam potencial de influenciar a QVRS deste grupo populacional.

Apesar das informações relevantes apresentadas pelo presente estudo, deve-se ressaltar algumas de suas limitações. Primeiramente, trata-se de um estudo transversal e esse delineamento não permite estabelecer relações de causalidades, apenas associações. Além disso, apenas indivíduos da cidade de Belo Horizonte foram incluídos. É possível que outros resultados fosse encontrados caso este estudo fosse realizado em uma cidade brasileira com características distintas da cidade de Belo Horizonte. Portanto, os resultados do presente estudo não devem ser generalizados.

Cabe ressaltar ainda, que não foram incluídos indivíduos com déficits cognitivos ou de linguagem, pois tal fato implicaria na consistência interna dos dados obtidos, contudo, seria de extrema importância analisar a relação do ambiente com a funcionalidade e incapacidade desses indivíduos. Além disso, destacamos que o MQE, embora seja um instrumento válido para analisar o ambiente e contemple todos os domínios da CIF sobre Fatores Ambientais, não abrange todas as categorias e conceitos abarcados na CIF sobre Fatores Ambientais e, este fato pode representar uma limitação deste estudo.

Apesar dessas limitações, o atual estudo permite observar associações significantes entre o ambiente considerado como obstáculo e QVRS que corroboram com achados prévios da literatura. Esses resultados apontam para a necessidade de se considerar os fatores ambientais, principalmente aqueles que são obstáculos, em abordagens direcionadas à QVRS desses indivíduos.

São necessários mais estudos para melhor explorar a associação entre fatores ambientais e QVRS no intuito de conhecer o que pode ser modificado no ambiente físico e social para proporcionar melhor QVRS após AVC. Salienta-se ainda, que estes achados poderão ser úteis futuramente para comparação do impacto do ambiente no AVC e em diferentes populações.

\section{CONCLUSÃO}

Os fatores ambientais considerados como obstáculos e a QVRS possuem associação significante e negativa, ainda que seja de fraca magnitude.

Dentre os domínios específicos da QVRS, apenas mobilidade e trabalho/produtividade se correlacionaram significativamente com ambiente obstáculo. Essas associações podem auxiliar em estudos futuros relacionados com a QVRS de indivíduos na fase crônica após AVC usuários da atenção primária à saúde.

É necessário, porém, mais estudos no intuito de melhor explorar a associação entre fatores ambientais e qualidade de vida em indivíduos da atenção primária do SUS, principalmente considerando outros contextos, como aqueles que são distintos dos observados na cidade de Belo Horizonte. Estudos envolvendo a associação de itens específicos dos fatores ambientais com os domínios da QVRS poderiam auxiliar em estratégias futuras a serem realizadas na atenção primária no intuito de melhor atender os usuários após AVC.

\section{REFERÊNCIAS}

1. World Health Organization. WHO STEPS Stroke Manual: the WHO STEPwise approach to stroke surveillance. Geneva: WHO; 2016. 
2. Bensenor IM, Goulart AC, Szwarcwald CL, Vieira ML, Malta DC, Lotufo PA. Prevalence of stroke and associated disability in Brazil: National Health Survey--2013. Arq Neuropsiquiatr. 2015;73(9):746-50. DOI: http://dx.doi.org/10.1590/0004282X20150115

3. Carvalho-Pinto BPB, Faria CDCM. Health, function and disability in stroke patients in the community. Braz J Phys Ther. 2016; 20(4):355-66. DOI: http://dx.doi.org/10.1590/bjpt-rbf.2014.0171

4. Brasil. Ministério da Saúde. Diretrizes de atenção à reabilitação da pessoa com acidente vascular cerebral. Brasília (DF): Ministério da Saúde; 2013.

5. CIF: Classificação Internacional de Funcionalidade, Incapacidade e Saúde. São Paulo: Edusp; 2003.

6. Engel L, Chudyk AM, Ashe MC, McKay HA, Whitehurst DGT, Bryan S. Older adults' quality of life - Exploring the role of the built environment and social cohesion in community-dwelling seniors on low income. Soc Sci Med. 2016;164:1-11. DOI: http://dx.doi.org/10.1016/j.socscimed.2016.07.008

7. Ferrans CE, Zerwic JJ, Wilbur JE, Larson JL. Conceptual model of health-related quality of life. J Nurs Scholarsh. 2005;37(4):336-42. DOI: http://dx.doi.org/10.1111/j.1547-5069.2005.00058.x

8. Leidy NK, Revicki DA, Genesté B. Recommendations for evaluating the validity of quality of life claims for labeling and promotion. Value Health. 1999;2(2):113-27. DOI http://dx.doi.org/10.1046/j.1524-4733.1999.02210.x

9. Algurén B, Lundgren-Nilsson A, Sunnerhagen KS. Facilitators and barriers of stroke survivors in the early post-stroke phase. Disabil Rehabil. 2009;31(19):1584-91. http://dx.doi.org/10.1080/09638280802639004

10. Colver AF, Dickinson HO; SPARCLE group. Study protocol: determinants of participation and quality of life of adolescents with cerebral palsy: a longitudinal study (SPARCLE2). BMC Public Health. 2010;10:280. DOI: http://dx.doi.org/10.1186/1471-245810-280

11. Foley KR, Girdler S, Bourke J, Jacoby P, Llewellyn G, Einfeld S, et al. Influence of the environment on participation in social roles for young adults with down syndrome. PLoS One. 2014;9(9):e108413. DOI: http://dx.doi.org/10.1371/journal.pone.0108413

12. Derguy C, Roux S, Portex M, M'bailara K. An ecological exploration of individual, family, and environmental contributions to parental quality of life in autism. Psychiatry Res. 2018;268:87-93. DOI: http://dx.doi.org/10.1016/j.psychres.2018.07.006

13. Brasil. Ministério da Saúde, Conselho Nacional de Saúde. Resolução n. 452, de 10 de maio de 2012. Resolve que a Classificação Internacional de Funcionalidade, Incapacidade e Saúde - CIF seja utilizada no Sistema Único de Saúde, inclusive na Saúde Suplementar. Diário Oficial da Republica Federativa do Brasil, Brasília (DF); 2012 Jun 06; Seção 1:137-8.

14. Brasil. Portaria n. 665, de 12 de abril de 2012. Dispõe sobre os critérios de habilitação dos estabelecimentos hospitalares como Centro de Atendimento de Urgência aos Pacientes com Acidente Vascular Cerebral (AVC), no âmbito do Sistema Único de Saúde (SUS), institui o respectivo incentivo financeiro e aprova a Linha de Cuidados em AVC. Diário Oficial da Republica Federativa do Brasil, Brasília (DF); 2012 Abr 13; Seção 1:35.

15. Brasil. Portaria n. 154, de 24 de janeiro de 2008. Cria os Núcleos de Apoio à Saúde da Família-NASF. Diário Oficial da Republica Federativa do Brasil, Brasília (DF); 2008 Mar 04; Seção 1:38-42.

16. Williams LS, Weinberger M, Harris LE, Biller J. Measuring quality of life in a way that is meaningful to stroke patients. Neurology. 1999;53(8):1839-43.

http://dx.doi.org/10.1212/wnl.53.8.1839

17. Duncan PW, Jorgensen HS, Wade DT. Outcome measures in acute stroke trials: a systematic review and some recommendations to improve practice. Stroke. 2000;31(6):1429-38. DOI: http://dx.doi.org/10.1161/01.str.31.6.1429
18. Lima RCM, Teixeira-Salmela LF, Magalhães LC, Gomes-Neto MV. Propriedades psicométricas da versão brasileira da escala de qualidade de vida específica para acidente vascular encefálico: aplicação do modelo Rasch. Rev Bras Fisioter. 2008;12(2):149-56. DOI: http://dx.doi.org/10.1590/S1413-35552008000200012

19. Fougeyrollas $P$, Noreau L, St-Michel G, Boschen K. Measure of the quality - version 2.0. Quebec: International Network of Disability Creation Process; 1999.

20. Alvarelhão J, Silva A, Martins A, Queirós A, Amaro A, Rocha N, et al. Comparing the content of instruments assessing environmental factors using the International Classification of Functioning, Disability and Health. J Rehabil Med. 2012;44(1):1-6. DOI: http://dx.doi.org/10.2340/16501977-0905

21. Faria-Fortini I, Basílio ML, Assumpção FSN, Teixeira-Salmela LF. Adaptação transcultural e reprodutibilidade do Measure of the Quality of the Enviroment em indivíduos com hemiparesia. Rev Ter Ocup Univ São Paulo. 2016;27(1):42-51. DOI: https://doi.org/10.11606/issn.2238-6149.v27i1p42-51

22. Campbell A, Hemsley S. Outcome Rating Scale and Session Rating Scale in psychological practice: clinical utility of ultra-brief measures. Clin Psychol. 2009;13(1):1-9. Doi: http://dx.doi.org/10.1080/13284200802676391

23. Fugl-Meyer AR, Jääskö L, Leyman I, Olsson S, Steglind S. The poststroke hemiplegic patient. 1. a method for evaluation of physical performance. Scand J Rehabil Med. 1975;7(1):13-31.

24. Michaelsen SM, Rocha AS, Knabben RJ, Rodrigues LP, Fernandes CGC. Tradução, adaptação e confiabilidade interexaminadores do manual de administração da escala de Fugl-Meyer. Rev Bras Fisioter. 2011;15(1):80-8. DOI: http://dx.doi.org/10.1590/S141335552011000100013

25. Dancey CP, Reidy J. Estatística sem matemática para psicologia: usando SPSS para Windows. Porto Alegre: Artmed; 2006.

26. Howe MD, McCullough LD. Prevention and management of stroke in women. Expert Rev Cardiovasc Ther. 2015;13(4):403-15. DOI: http://dx.doi.org/10.1586/14779072.2015.1020300

27. Mozaffarian D, Benjamin EJ, Go AS, Arnett DK, Blaha MJ, Cushman $M$, et al. Heart disease and stroke statistics-2016 update: a report from the American Heart Association. Circulation. 2016;133(4):e38-360. http://dx.doi.org/10.1161/CIR.0000000000000350

28. Copstein L, Fernandes JG, Bastos GA. Prevalence and risk factors for stroke in a population of Southern Brazil. Arq Neuropsiquiatr. 2013;71(5):294-300. http://dx.doi.org/10.1590/0004 $282 \times 20130024$

29. Dupre ME. Race, rarital history, and risks for stroke in US older adults. Soc Forces. 2016;95(1):439-468. DOI: http://dx.doi.org/10.1093/sf/sow040

30. Honjo K, Iso H, Ikeda A, Inoue M, Sawada N, Tsugane S. Marital transition and risk of stroke: how living arrangement and employment status modify associations. Stroke. 2016;47(4):991-8. DOI: http://dx.doi.org/10.1161/STROKEAHA.115.011926

31. Addo J, Ayerbe L, Mohan KM, Crichton S, Sheldenkar A, Chen R, et al. Socioeconomic status and stroke: an updated review. Stroke. 2012;43(4):1186-91.

DOI: http://dx.doi.org/10.1161/STROKEAHA.111.639732

32. McHutchison CA, Backhouse EV, Cvoro V, Shenkin SD, Wardlaw JM. Education, socioeconomic status, and intelligence in childhood and stroke risk in later life: a meta-analysis. Epidemiology. 2017;28(4):608-618. http://dx.doi.org/10.1097/EDE.0000000000000675

33. Gallacher KI, Batty GD, McLean G, Mercer SW, Guthrie B, May CR, et al. Stroke, multimorbidity and polypharmacy in a nationally representative sample of $1,424,378$ patients in Scotland: implications for treatment burden. BMC Med. 2014;12:151. DOI: http://dx.doi.org/10.1186/s12916-014-0151-0 
34. Gruneir A, Griffith LE, Fisher K, Panjwani D, Gandhi S, Sheng L, et al. Increasing comorbidity and health services utilization in older adults with prior stroke. Neurology. 2016;87(20):2091-2098. DOI: http://dx.doi.org/10.1212/WNL.0000000000003329

35. Langhorne $P$, Coupar F, Pollock A. Motor recovery after stroke: a systematic review. Lancet Neurol. 2009;8(8):741-54. DOI: http://dx.doi.org/10.1016/S1474-4422(09)70150-4

36. Raghavan P. Upper limb motor impairment after stroke. Phys Med Rehabil Clin N Am. 2015;26(4):599-610. DOI: http://dx.doi.org/10.1016/j.pmr.2015.06.008

37. Pereira CAR, Melo JV, Fernandes ALT. A educação ambiental como estratégia da Atenção Primária à Saúde. Rev Bras Med Fam Comunidade. 2012;7(23):108-16.

DOI: http://dx.doi.org/10.5712/rbmfc7(23)293

38. Parra DC, Gomez LF, Sarmiento OL, Buchner D, Brownson R, Schimd $T$, et al. Perceived and objective neighborhood environment attributes and health related quality of life among the elderly in Bogotá, Colombia. Soc Sci Med. 2010;70(7):1070-6. DOI: http://dx.doi.org/10.1016/j.socscimed.2009.12.024

39. Badia M, Begoña Orgaz M, Gómez-Vela M, Verdugo MA, Ullán AM, Longo $E$. Do environmental barriers affect the parent-reported quality of life of children and adolescents with cerebral palsy? Res Dev Disabil. 2016;49-50:312-21. DOI: http://dx.doi.org/10.1016/j.ridd.2015.12.011

40. Law M, Hanna S, Anaby D, Kertoy M, King G, Xu L. Health-related quality of life of children with physical disabilities: a longitudinal study. BMC Pediatr. 2014;14:26. DOI: http://dx.doi.org/10.1186/1471-2431-14-26

41. Yeung $P$, Towers A. An exploratory study examining the relationships between the personal, environmental and activity participation variables and quality of life among young adults with disabilities. Disabil Rehabil. 2014;36(1):63-73. DOI: http://dx.doi.org/10.3109/09638288.2013.777808
42. Zhao Y, Chung PK. Neighborhood environment walkability and health-related quality of life among older adults in Hong Kong. Arch Gerontol Geriatr. 2017;73:182-186. DOI: http://dx.doi.org/10.1016/j.archger.2017.08.003

43. Gobbens RJJ, van Assen MALM. Associations of Environmental Factors With Quality of Life in Older Adults. Gerontologist. 2018;58(1):101-110.

http://dx.doi.org/10.1093/geront/gnx051

44. Canuto MAO, Nogueira LT, Araujo TME. Qualidade de vida relacionada à saúde de pessoas após acidente vascular cerebral. Acta Paul Enferm. 2016;29(3):245-52. DOI: http://dx.doi.org/10.1590/1982-0194201600035

45. Schmid AA, Van Puymbroeck M, Altenburger PA, Miller KK, Combs SA, Page SJ. Balance is associated with quality of life in chronic stroke. Top Stroke Rehabil. 2013;20(4):340-6. DOI: http://dx.doi.org/10.1310/tsr2004-340

46. Riberto M, Lopes KA, Chiappetta LM, Lourenção MI, Battistella LR. The use of the comprehensive International Classification of Functioning, Disability and Health core set for stroke for chronic outpatients in three Brazilian rehabilitation facilities. Disabil Rehabil. 2013;35(5):367-74. DOI: http://dx.doi.org/10.3109/09638288.2012.694573

47. Starfield B. Atenção primária: equilíbrio entre necessidades de saúde, serviços e tecnologia. Brasília (DF): Unesco, Ministério da Saúde; 2002.

48. Ascef BO, Haddad JPA, Álvares J, Guerra AA Junior, Costa EA, Acurcio FA, et al. Health-related quality of life of patients of Brazilian primary health care. Rev Saude Publica. 2017;51(suppl 2):22s. DOI: http://dx.doi.org/10.11606/S1518- 